\title{
Histological, Physical Studies after Xenograft of Porcine Ear Cartilage
}

Yong Ah Ryu,

Meiying Jin, Nakheon Kang

Department of Plastic and Reconstructive Surgery, Chungnam National University School of Medicine, Daejeon, Korea

No potential conflict of interest relevant to this article was reported.

\begin{abstract}
Background: Because of the relatively similar size of organs to human and the physiological and structural similarities, the use of porcine as xenograft donors is progressing very actively. In this study, we analyzed the characteristics of porcine ear cartilage and evaluated its suitability as graft material in reconstructive and cosmetic surgery.

Methods: The auricular cartilage was harvested from two pigs, and subjected to histological examination by immunohistochemical staining. To determine the collagen content, samples were treated with collagenase and weight changes were measured. After sterilization by irradiation, the samples were grafted into rats and stained with Hematoxylin and Eosin and Masson Trichrome to observe inflammation and xenograft rejection.

Results: In IHC staining, extracellular matrices were mainly stained with type II collagen $(20.69 \%)$, keratin sulfate $(10.20 \%)$, chondroitin sulfate $(2.62 \%)$, and hyaluronic acid $(0.84 \%)$. After collagenase treatment, the weight decreased by $68.3 \%$, indicating that about $70 \%$ of the porcine ear cartilage was composed of collagen. Upon xenograft of the sterilized cartilages in rats, inflammatory cells were observed for up to 2 months. However, they gradually decreased, and inflammation and reject-response were rarely observed at 5 months.

Conclusion: The porcine ear cartilage was covered with perichondrium and cellular constituents were found to be composed of chondrocytes and chondroblasts. In addition, the extracellular matrices were mainly composed of collagen. Upon xenograft of irradiated cartilage into rats, there was no specific inflammatory reaction around the transplanted cartilage. These findings suggest that porcine ear cartilage could be a useful alternative implant material for human cosmetic surgery.
\end{abstract}

Keywords: Ear cartilage / Swine / Xenograft

\section{INTRODUCTION}

Autogenous cartilage is the most commonly used graft material in the reconstruction of congenital auricular anomalies and aesthetic rhinoplasties. Even though autologous cartilage is less infectious and causes fewer side effects than alloplastic material, the amount of autologous cartilage that can be harvested is limited [1]. To overcome this disadvantage, the homogenous cartilage ob-

\section{Correspondence: Nakheon Kang}

Department of Plastic and Reconstructive Surgery, Chungnam National University

Hospital, 282 Munhwa-ro, Jung-gu, Daejeon 35015, Korea

E-mail:nhk488@cnu.ac.kr

Received May 26, 2017 / Revised August 29, 2017 / Accepted August 29, 2017 tained from a human cadaver is used; however, this is expensive and it is difficult to obtain sufficient quantities of cartilage [2]. Because of a lack of supply and high cost, research on xenografts is progressing actively. Among potential xenograft donors, pigs have shorter gestation periods and grow faster. More importantly, pig organs are similar to human organs in size, anatomy, and physiology. In addition, it is easy to obtain pathogen-free pigs with fewer xenozoonoses, and the blood type compatibility is relatively insignificant. Therefore, porcine xenotransplantation studies of cells, tissues, and organs have been actively conducted. There are various types of transplantations: (1) cellular transplantation using islet cell, neuronal cell, chromaffin cell, hepatocyte, and cornea; and 
(2) organ/tissue transplantations using heart valve, vessel, lung, liver, kidney, and heart. In addition, studies have investigated the treatment of acute and chronic wounds and bone fragment transplantation using porcine skin and small intestine submucosa [3].

Among the various porcine tissues, cartilage tissue has been of particular interest because of its potential for treatment of degenerative arthritis and reconstruction of the tracheal defects. Animal experiments have been conducted to collect chondrocytes and cultivate cartilage tissue from pigs. However, it takes a long time to cultivate these cells, and there are side effects associated with transplantation. All these challenges make use of porcine tissues in clinical practice difficult. As such, few studies have investigated the use of xenografts in aesthetic rhinoplasty or reconstruction of ear deformity.

In this study, the authors examined the possibility of xenotransplantation using porcine cartilage for cosmetic and reconstructive surgery by examining histological analyses and physical properties of the porcine cartilage. Additionally, after irradiation with gamma rays and sterilization, xenografts were into the rats were performed to study the tissue responses.

\section{METHODS}

\section{Materials}

All experimental procedures were carried out according to the regulations of the Animal Experiment Ethics Committee of Chungnam National University. The ear cartilage of pigs was collected from two pigs that were 6 months of age. Additionally, 10 Sprague-Dawley (SD) rats weighing 250 to $350 \mathrm{~g}$ were used for xenotransplantation at 4 weeks of age.

\section{Experimental Methods}

\section{Histological analysis (immunohistochemical stain)}

A $4 \mu \mathrm{m}$ thick paraffin section fixed to formalin was deparaffinized by reacting with a HistoClear II solution (Fisher Scientific, Pittsburgh, PA, USA) for 20 minutes, followed by rehydration with graded ethanol solutions $(100 \%, 90 \%, 70 \%)$ for 2 minutes each. The cartilaginous tissue was then treated with $3.0 \%$ hydrogen peroxidase solution for 10 minutes to inhibit the endogenous enzyme. Proteinase K (Sigma, St. Louis, MO, USA) pretreatment was performed for 15 minutes to release the cross-linking formed by formalin fixation and increase antigen exposure, after which the tissue was treated with 10X SuperBlock solution (Pierce, Rockford, IL, USA) for 30 minutes to inhibit proteins that could specifically bind to the primary antibody. The primary antibodies used were as follows: anti-keratin sulfate IgG (1:100, sc-73518, Santa Cruz Biotechnology, Santa Cruz, CA, USA), anti-chondroitin sulfate $\operatorname{IgM}$ (1:200, ab11570, Abcam, Cambridge, MA, USA), anti-hyaluronic acid $\operatorname{IgG}(1: 50$, ab53842, Abcam), and anti-type II collagen ab-2 IgG (1:200, MS235PO, Thermo Scientific, Waltham, MA, USA). All antibodies reacted with tissue sections and incubated in a wet chamber. As secondary antibodies, anti-keratan sulfate antibody, anti-chondroitin sulfate antibody, and type II collagen Ab-2 antibody were used in EnVision complex (DAKO, Hamburg, Germany), while anti-hyaluronic acid antibody was used in rabbit anti-sheep IgG (HRP) (ab6747, Abcam, Cambridge, MA, USA). Additionally, NovaRED (Vector, Burlingame, CA, USA) was used as a chromogen and immersed in Mayer's hematoxylin (Sigma) for 7 minutes with counterstain. After dehydration and clearing, the samples were mounted on a slide and observed under a 200× optical microscope. After scanning the stained slides, slides were photographed and analyzed using the Image software (version 1.50i, National Institutes of Health, Bethesda, MD, USA).

\section{Quantitative analysis of collagen}

Ten specimens each $0.5 \times 0.5 \mathrm{~cm}$ in size were prepared from the ears of two pigs. Collagenase at a concentration of $50 \mathrm{U} / \mathrm{mL}$ was added to each sample and stored at $37^{\circ} \mathrm{C}$ in an incubator. The degree of decomposition by collagenase was then determined based on the change in weight of the sample over a time interval of 7 days, and the amount of weight change evaluated using the Mann-Whitney test.

\section{Xenografting}

Porcine auricular cartilage was cut into $1 \times 1 \mathrm{~cm}$ pieces and sterilized by irradiation with gamma radiation at $25 \mathrm{kGy}$ using a Cobalt- 60 irradiator (IR-149 irradiator, 60Co panel source) [4]. Decellulariza- 
tion was performed by immersing a cartilage specimen into a PBS solution containing $0.5 \mathrm{mg} / \mathrm{mL}$ DNases Type I, $50 \mu \mathrm{g} / \mathrm{mL}$ RNase, 0.02 EDTA and $1 \% \mathrm{P} / \mathrm{S} / \mathrm{F}$ according to the method described by Elder et al. [5]. Next, 2\% sodium dodecyl sulfate (SDS) solution was added and reacted for 8 hours [6]. Xenografts of the porcine auricular cartilage were subsequently implanted between the skin and muscle layer on the left and right sides of 10 male SD rats that weighed 250-350 g. Histologically, H\&E and Masson trichrome (MT) stain were obtained after the rats were sacrificed monthly.

\section{RESULTS}

\section{Immunohistochemical (IHC) stain}

The type II collagen, which was stained the most (20.69\%) by IHC
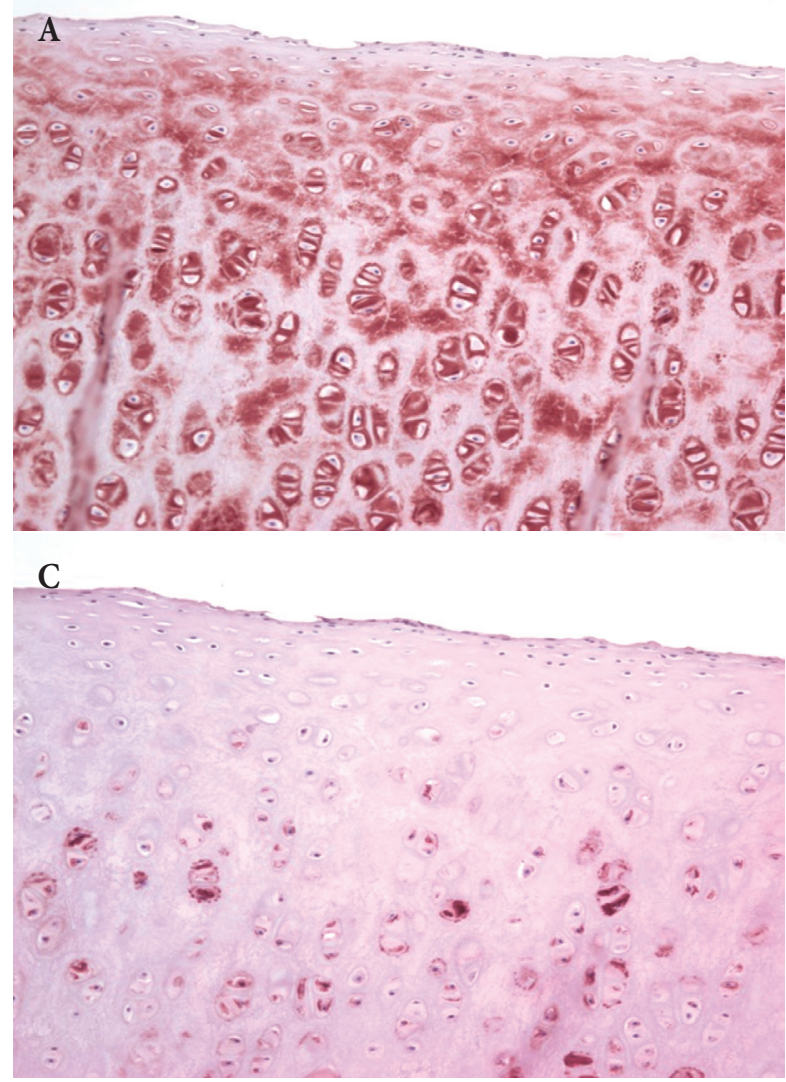

staining, was distributed throughout the extracellular matrix around the chondrocytes under the perichondrium. Among the ground substances, keratan sulfate was the second most abundant (10.20\%) and mainly distributed in the extracellular matrix around the mature chondrocytes in the deep layer below the perichondrium. The amount of chondroitin sulfate was the next highest (2.62\%), and was distributed mainly in the extracellular matrix on the outer edge around the immature chondrocytes. Hyaluronic acid was the least abundant (0.84\%). Although not distributed deep into the tissue, hyaluronic acid was relatively widely distributed (Fig. 1).

\section{Weight changes after collagenase treatment}

The weight decreased to $51.08 \%$ of the initial mass during the first

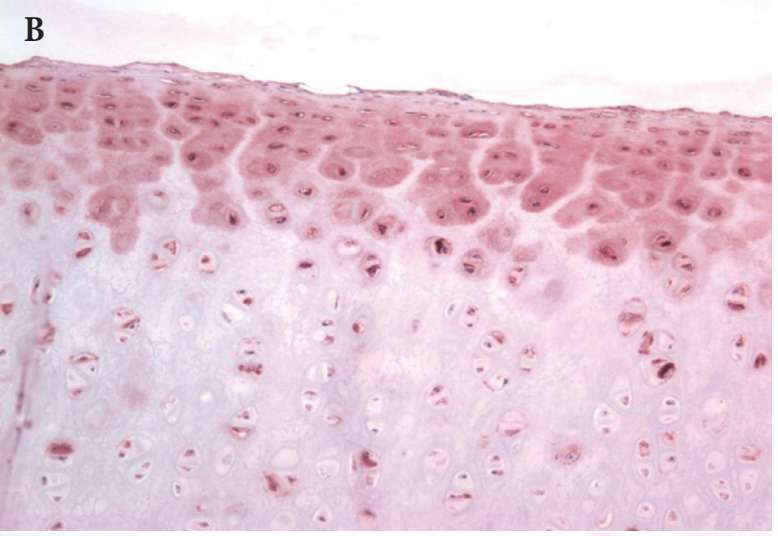

D

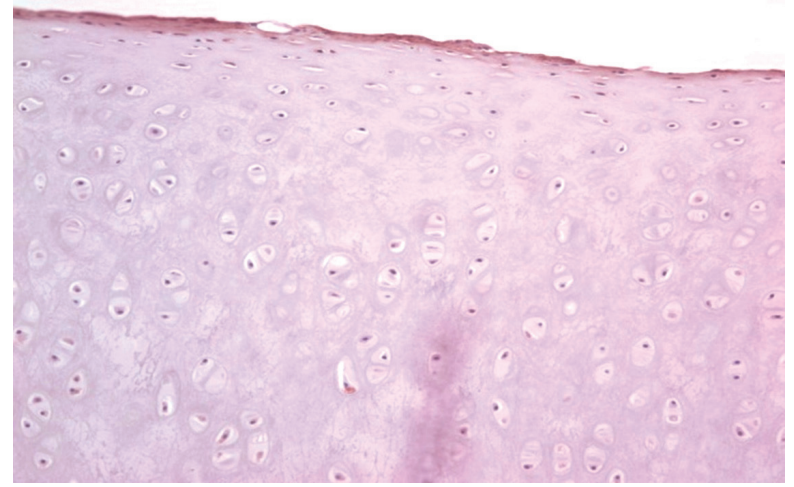

Fig. 1. Immunohistochemical staining $(\times 400)$. Type II collagen $(A)$ was evenly distributed in the extracellular matrix around chondrocytes below the perichondrium. Among the ground substances, keratan sulfate (B) was mainly distributed in the extracellular matrix around mature chondrocytes in the deep layer below the perichondrium. Chondroitin sulfate (C) was primarily distributed in the extracellular matrix around the outer edge of young chondrocytes. The amount of hyaluronic acid (D) was small and not stained densely, but distributed relatively widely. 
50 hours after collagenase treatment, then further decreased to $31.65 \%$ after about 7 days. There was little change in mass after 7 days. Therefore, the total amount of collagen was about $68.35 \%$ (Table 1, Fig. 2). The weight loss during the first 50 hours was $0.98 \%$ per hour, while the weight loss from 50 hours to 7 days was $0.16 \%$ per hour, which was statistically significant $(p<0.001)$ (Fig.

3). Thus, it was estimated that most of the porcine auricular cartilage was made up of collagen.

\section{Xenograft}

H\&E staining and MT staining were performed at 1,2, 3, 4, and 5 months after implantation of cartilage, and inflammatory cells were found around the transplanted cartilage for up to 2 months. However, they gradually decreased, and inflammation and rejectresponse were rarely found at 5 months, and the collagen in the

Table 1. Review of the 9 recurrent cases

\begin{tabular}{|cc|}
\hline Time $(\mathrm{hr})$ & Weight $(\%)$ \\
\hline 1 & 100 \\
\hline 5 & 95.56 \\
\hline 10 & 88.04 \\
\hline 15 & 76.34 \\
\hline 20 & 67.37 \\
\hline 25 & 62.26 \\
\hline 50 & 51.08 \\
\hline 168 & 31.65 \\
\hline
\end{tabular}

For the first 50 hours after being treated with collagenase, the weight was reduced by $51.08 \%$ and decreased by $31.6 \%$ after 7 days, after which there was no significant change in weight. Thus, the average collagen mass was about $68.35 \%$.

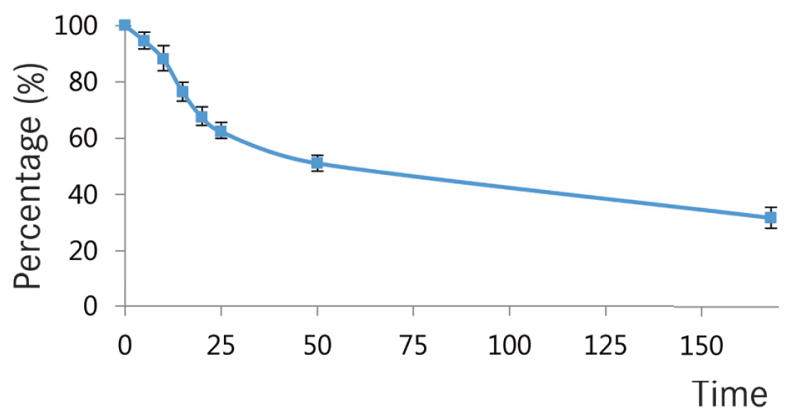

Fig. 2. Weight reduction after collagenase treatment.

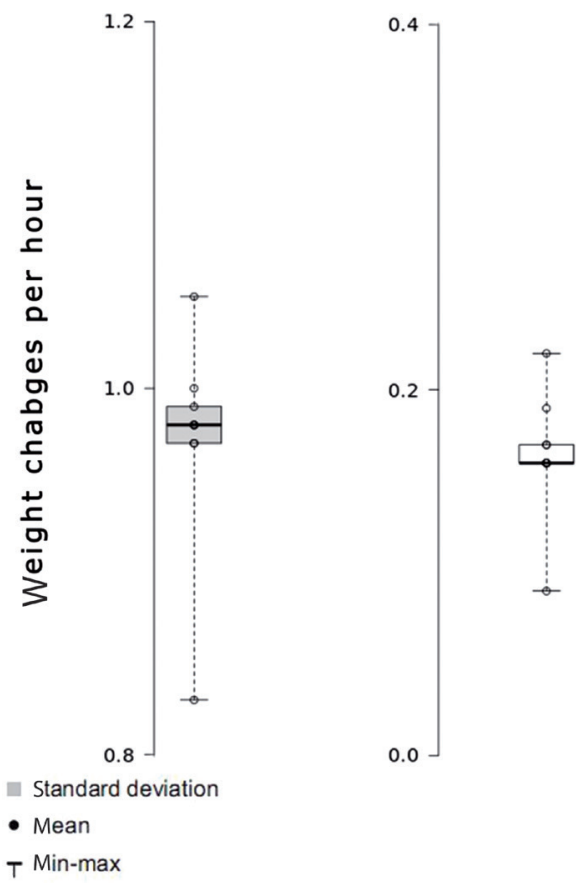

Fig. 3. Mean weight change per hour after collagenase treatment. The mean weight reduction per hour for the first 50 hours was $0.98 \%$, while it was $0.16 \%$ from 50 hours to 7 days. There was a statistically significant difference between groups $(p<0.01)$.

cartilage did not decrease over time, although calcification within the cartilage was observed 3 months later (Fig. 4).

\section{DISCUSSION}

In cosmetic and reconstructive surgery, both biomaterials (such as cartilage) and artificial materials (such as Medpor and silicone) are commonly used as implantable materials in clinical practice [7-9]. These graft materials are used for augmentation rhinoplasty and tip plasty in cosmetic surgery [10], and widely applied for reconstruction of congenital auricular anomalies such as microtia or in cases of traumatic ear amputation [11]. Artificial materials can easily be molded into the desired shape, and large amounts can be produced. However, they can induce various side effects such as inflammation and escape of the graft [12]. On the other hand, transplantation of autologous cartilage causes less inflammation. However, the donor sites of cartilage grafts are limited to septum cartilage, ear cartilage, and costal bone; thus, the amount 

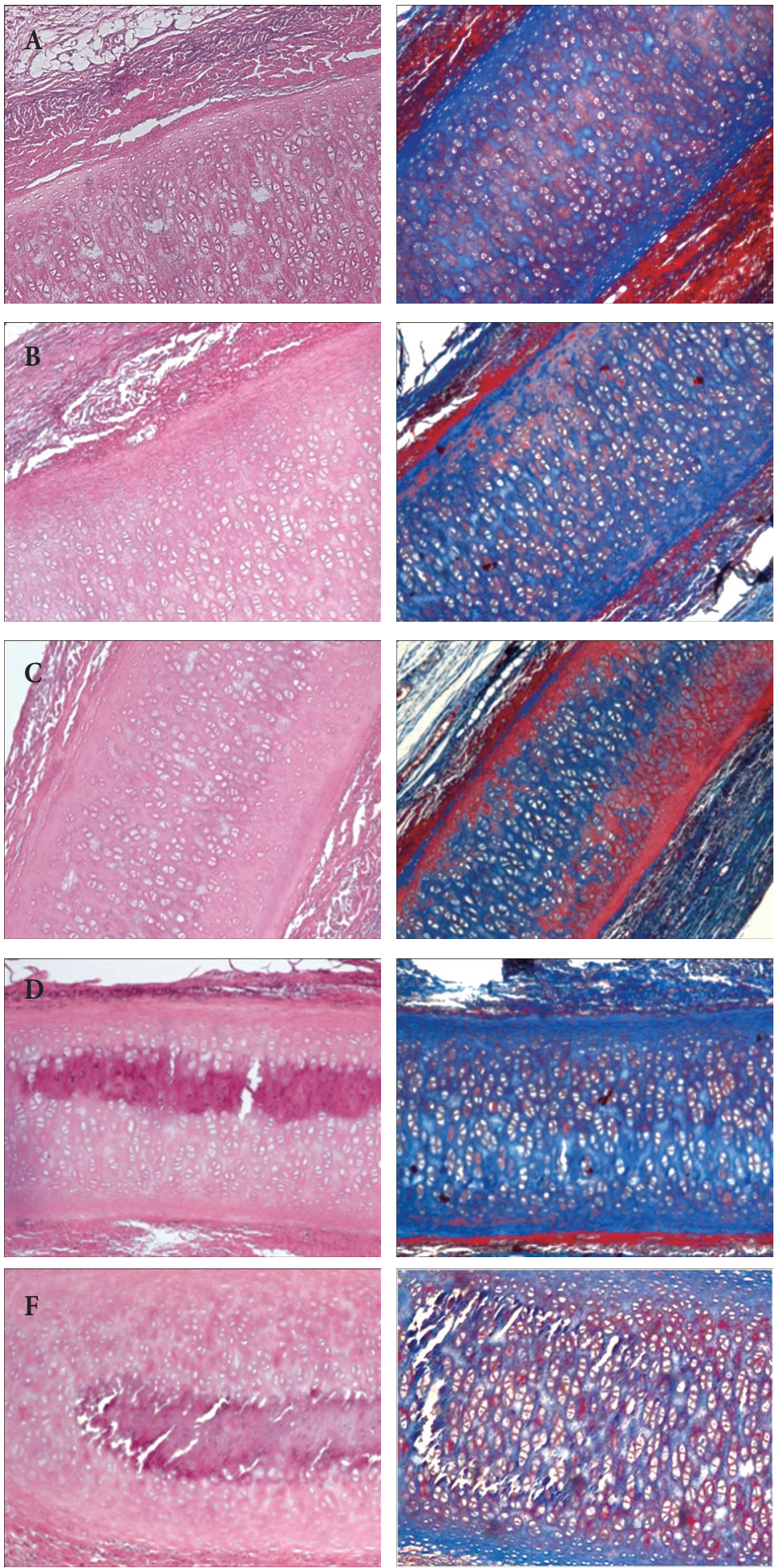

Fig. 4. Xenograft in the rat ( $\times 40)$. (A) H\&E, MT staining at 1 month after transplantation. Around the implanted cartilage, inflammatory cells (lymphocytes) and giant cells were observed in small quantities. (B) 2-month H\&E, MT stain. Lymphocytes were still observed, but in reduced amounts. (C) 3-month H\&E, MT stain. The inflammatory cells were further reduced and a small amount of calcification was observed in the grafted cartilage. (D) 4-month H\&E, MT stain. Inflammatory cells were not observed in substantial numbers, but calcification was increased. (E) 5-month H\&E, MT stain. Inflammatory cells were not observed, calcification was not increased and the volume of the cartilage was not altered. MT, Masson trichrome. 
available is limited. In addition, when collecting costal bones in large quantities at once, it may be necessary to reconstruct the donor defect [13]. Recently, human cadaver cartilage has been used as an implant for cosmetic and reconstructive surgery. This has fewer side effects than artificial materials, and there are few restrictions to obtain because there is no sacrifice of the donor site. However, this cartilage is expensive, and difficult to use in large quantities [14]. Accordingly, the authors investigated the elastic ear cartilage of pigs, which is known to have tissue structure similar to humans and is relatively inexpensive and readily available.

Elastic cartilage is composed of chondrocytes and extracellular matrix, and the extracellular matrix can be divided into fibrous and ground substances [15]. The fibers are mostly type II collagen and elastic fibers, whereas the ground substances are composed of glycosaminoglycans (GAGs) [16,17]. Cartilage can be further classified according to the composition and characteristics of the extracellular matrix. Hyaline cartilage, which is rich in extracellular matrix compared to cells, is present in the ribs, bronchi, and joints. Elastic cartilage, which has a lot of elastic fibers in the extracellular matrix, is present in the ear and ear canal, but the extracellular matrix is relatively small when compared to the hyaline cartilage [14]. Fibrous cartilage contains a large amount of type I collagen and is part of the intervertebral disk and joint [18].

In a preliminary study, H\&E staining showed that porcine auricular cartilage was covered by the perichondrium, which was composed of dense connective tissue that consisted of chondrocytes and an extracellular matrix. Fibrocytes and fibroblasts existed in the perichondrium, and chondroblasts were observed in the chondrogenic layer, which was below the perichondrium. In the deeper layer, there were lacunae containing chondroblasts and cell nests composed of two or four chondrocytes. Immunohistochemical staining revealed that keratan sulfate and type II collagen in the extracellular matrix were distributed evenly throughout the entire cartilage. Chondroitin sulfate was mainly distributed on the outer edge, while hyaluronic acid was distributed evenly although in small amounts. Furthermore, the extracellular matrix of the porcine ear cartilage contained GAGs such as type II collagen, keratan sulfate, chondroitin sulfate, and hyaluronic acid.
As a result of observing porcine cartilage specimens in collagenase solution, the amount of collagen decreased to $51.08 \%$ within the first 50 hours, then further decreased to $31.65 \%$ after 7 days. In other words, most of the pig's ear cartilage is made of collagen.

Following xenograft in SD rats, small amounts of inflammatory cells were observed around the grafts; however, these were rarely observed after 5 months. Therefore, it is expected that there will be no severe inflammatory reaction or rejection after gamma-ray sterilization and decellularization.

In the future, a series of experiments should be conducted to compare the elasticity, hardness, tensile strength, and bending strength of porcine ear cartilage with those of human ear cartilage.

Immunohistochemical staining revealed that the porcine ear cartilage was surrounded by the perichondrium, which consisted primarily of chondroblasts and chondrocytes. In the extracellular matrix, the fibrous component consists of type II collagen, whereas the ground substance was composed of keratan sulfate, chondroitin sulfate, and hyaluronic acid. Measurement of the weight change over time post-collagenase treatment revealed that the total amount of collagen decreased to $68.35 \%$, indicating that most of the cartilage tissue was collagen. Following xenografting of the sterilized porcine ear cartilages into rats, few inflammatory cells were observed after 2 months, and the cartilage size did not decrease. Therefore, no specific rejection or inflammation reaction was observed.

Studies of mechanical properties such as hardness, elasticity, tensile strength, and bending strength of porcine ear cartilage should be conducted to enable comparison with those of human ear cartilage. If additional studies further support our observations, the auricular cartilage of pig may be suitable for clinical use as a prosthetic implant.

\section{REFERENCES}

1. Tosun Z, Karabekmez FE, Keskin M, Duymaz A, Savaci N. Allogenous cartilage graft versus autogenous cartilage graft in augmentation rhinoplasty: a decade of clinical experience. Aesthetic Plast Surg 2008;32:252-60.

2. Kridel RW, Ashoori F, Liu ES, Hart CG. Long-term use and follow-up 
of irradiated homologous costal cartilage grafts in the nose. Arch Facial Plast Surg 2009;11:378-94.

3. Cooper DK. A brief history of cross-species organ transplantation. Proc (Bayl Univ Med Cent) 2012;25:49-57.

4. Shintani $\mathrm{H}$. The relative safety of gamma-ray, autoclave, and ethylene oxide gas sterilization of thermosetting polyurethane. Biomed Instrum Technol 1995;29:513-9.

5. Elder BD, Eleswarapu SV, Athanasiou KA. Extraction techniques for the decellularization of tissue engineered articular cartilage constructs. Biomaterials 2009;30:3749-56.

6. Gilbert TW, Sellaro TL, Badylak SF. Decellularization of tissues and organs. Biomaterials 2006;27:3675-83.

7. Maas CS, Monhian N, Shah SB. Implants in rhinoplasty. Facial Plast Surg 1997;13:279-90.

8. Reiffel AJ, Cross KJ, Spinelli HM. Nasal spreader grafts: a comparison of medpor to autologous tissue reconstruction. Ann Plast Surg 2011; 66:24-8.

9. Ahn J, Honrado C, Horn C. Combined silicone and cartilage implants: augmentation rhinoplasty in Asian patients. Arch Facial Plast Surg 2004;6:120-3.

10. Ortiz-Monasterio F, Olmedo A, Oscoy LO. The use of cartilage grafts in primary aesthetic rhinoplasty. Plast Reconstr Surg 1981;67:597-605.

11. Brent B, Byrd HS. Secondary ear reconstruction with cartilage grafts covered by axial, random, and free flaps of temporoparietal fascia. Plast Reconstr Surg 1983;72:141-52.

12. Peled ZM, Warren AG, Johnston P, Yaremchuk MJ. The use of alloplastic materials in rhinoplasty surgery: a meta-analysis. Plast Reconstr Surg 2008;121:85e-92e.

13. Gryskiewicz JM. Waste not, want not: the use of AlloDerm in secondary rhinoplasty. Plast Reconstr Surg 2005;116:1999-2004.

14. Komender J, Malczewska H, Sladowski D, Komender A. Allogenic preserved costal cartilage in reconstructive surgery. Ann Transplant 1997;2:49-51

15. Bian W, Lian Q, Li D, Wang J, Zhang W, Jin Z, et al. Morphological characteristics of cartilage-bone transitional structures in the human knee joint and CAD design of an osteochondral scaffold. BioMedical Engineering OnLine 2016;15:82.

16. DePalma RL, Krummel TM, Durham LA, 3rd, Michna BA, Thomas BL, Nelson JM, et al. Characterization and quantitation of wound matrix in the fetal rabbit. Matrix 1989;9:224-31.

17. Mankin HJ, Lippiello L. The glycosaminoglycans of normal and arthritic cartilage. J Clin Invest 1971;50:1712-9.

18. Cardenas-Camarena L, Gomez RB, Guerrero MT, Solis M, Guerrerosantos J. Cartilaginous behavior in nasal surgery: a comparative observational study. Ann Plast Surg 1998;40:34-8. 